\title{
ESTILOS DE APRENDIZAJE IDENTIFICADOS EN DISEÑO INDUSTRIAL Y SU RELACIÓN CON LA FORMACIÓN AUTÓNOMA.
}

\author{
Luna, Carlos ${ }^{1}$, Forero Sandra ${ }^{1}$.
}

1 Diseñador Industrial, Especialista en Pedagogía Universitaria, Magister en Desarrollo Sustentable, Universidad de Pamplona, Docente auxiliar, Pamplona, Norte de Santander, pampluna@yahoo.com

1 Diseñadora Industrial, Especialista en Gestión de proyectos, Magister en Educación, Universidad de Pamplona, Docente asistente, Pamplona, Norte de Santander.

\section{RESUMEN}

El presente artículo permite evidenciar la relación entre los estilos de aprendizaje, el enfoque de aprendizaje autónomo y las estrategias de aprendizaje implementadas al interior del Programa de Diseño Industrial de la Universidad de Pamplona, basado en un estudio de corte transversal a través de datos recolectados con un instrumento tipo encuesta, con variables cualitativas analizadas estadísticamente.

Entre los resultados más representativos está la tendencia de los estudiantes por los Estilos Reflexivo y Teórico, los que al contrastar con la bibliografía revisada, se relacionan directamente con los métodos de enseñanza utilizados al interior del programa, y que dejan entrever la necesidad de reforzar el desarrollo de los estilos complementarios.

PALABRAS CLAVE: Estilos de aprendizaje, diseño industrial, aprendizaje autónomo, educación basada en proyectos, proceso enseñanza-aprendizaje.

\section{LEARNING STYLES IDENTIFIED IN INDUSTRIAL DESIGN AND ITS RELATIONSHIP WITH THE AUTONOMOUS TRAINING.}

\begin{abstract}
This article demonstrates the link between learning styles, the focus of autonomous learning and learning strategies implemented in the Industrial Design Program of the Universidad de Pamplona, based on a cross sectional study of data collected through with one survey instrument type, with qualitative variables statistically analyzed. Among the most representative results is the tendency of students by Reflective and Theoretical Styles, which to contrast with the literature reviewed, are directly related to the teaching methods used within the program, and which show the need to strengthen the development of complementary styles.
\end{abstract}

KEYWORDS: Learning styles, industrial design, independent learning, project-based education teaching-learning process. 


\section{INTRODUCCIÓN}

El fenómeno de la globalización obliga a la educación superior a replantear el objetivo de la formación profesional ya que principalmente las tecnologías de la información (entre otros muchos factores) han modificado el acceso al conocimiento, convirtiéndose este en formar para la autonomía, centrándose en: enseñar a aprender. Según León Guerrero (2004) "se trata de desarrollar el talento humano mediante la argumentación, la interpretación y la proposición; así el estudiante adquiere la capacidad de generar propuestas y acciones para la solución de problemas". Dejando a un lado la educación transmisionista, memorística y repetitiva.

Estos planteamientos han empezado a generar cuestionamientos y cambios en los escenarios de la educación superior, obligando a realizar un giro en el rol docente; lo resaltan diversos investigadores que como Álvarez y Moreno Marto (2006u) quienes afirman que "El profesor deberá pasar, ... a relegar las tareas docentes en relación con la transmisión de informaciones, dejar de ser docente informador, para pasar a ser docente formador; dejará de enseñar conocimientos para pasar a enseñar a aprender", o como lo expone también Rubia, A. y Marbán, J. $M^{a}$ (2006), resaltando que ya no es suficiente con los saberes tradicionales, más aún si se tiene la meta de formar personas "capaces de generar aprendizajes autónomos".

En el contexto Colombiano, se puede evidenciar que estos planteamientos no son del todo ajenos, según el Ministerio de Educación Nacional (MEN), la educación se define como "un proceso de formación permanente, personal, cultural y social que se fundamenta en una concepción integral de la persona humana, de su dignidad, de sus derechos y de sus deberes". Aspectos básicos para la formación autónoma, tal y como lo resalta lafrancesco (2012):

La tendencia educativa del último siglo, es desarrollar las competencias cognitivas, de la mano del adecuado desarrollo humano que permita a las futuras generaciones responder a las necesidades socioculturales de su momento. Dichas competencias involucran el saber hacer con el saber actuar, con el saber ser y con el saber pensar.

Este último autor, además concluye, que para alcanzar el aprendizaje autónomo se deben desarrollar en los educandos las operaciones mentales intelectivas, múltiples inteligencias y su potencial de aprendizaje. Sin embargo desde la experiencia del aula se percibe que este planteamiento sigue siendo un proceso de aciertos y fracasos mediados por estrategias y didácticas comunes a todo el estudiantado, desconociendo teorías ya comprobadas pero no tan comúnmente aplicadas en las didácticas de aula, entre ellas los estilos de aprendizaje de los educandos, cuyo diagnóstico o caracterización facilitan tanto a este como al docente la apropiación significativa de los conocimientos impartidos.

Para respaldar este aspecto se estudió y aplicó el enfoque propuesto por Alonso, Gallego y Honey (2008) quienes afirman que para que los estudiantes aprendan a aprender se debe hacer un uso eficaz y eficiente de los estilos de aprendizaje, entendidos como la forma o manera de aprender propia de un individuo es decir "los rasgos cognitivos, afectivos y fisiológicos, que sirven como indicadores relativamente estables, de cómo los estudiantes perciben, interaccionan y responden a sus ambientes de aprendizaje". 
Dicha caracterización permite clasificar las predominancias en cuatro categorías: activos, reflexivos, teóricos y pragmáticos, es importante resaltar que esta categorización plantea el aprendizaje como un modelo cíclico, donde se reúne la información, se analiza la documentación, se estructura y sintetiza dicha información y finalmente se lleva a la práctica todo lo elaborado; lo cual encaja perfectamente con el enfoque de aprendizaje autónomo, buscado en esta investigación.

Es indispensable entonces, entender que para una correcta apropiación del conocimiento se tiene que tener en cuenta el estilo de aprendizaje del educando, que en algunas ocasiones se ve relegado por el estilo de enseñanza propio del docente $u$ orientador.

Estos aspectos hacen parte del presente artículo, que pretende exponer, basado en los resultados de un proyecto de investigación desarrollado al interior del Programa de Diseño Industrial de la Universidad de Pamplona la relación entre los estilos de aprendizaje más representativos 0 preferidos por los estudiantes y su predisposición a ellos dada por el modelo de enseñanza.

\section{MÉTODOS}

Para la presente investigación se desarrolló un estudio descriptivo de carácter cuantitativo, con variables cualitativas, de corte transversal a través de la aplicación de un instrumento general tipo cuestionario, compuesto por 4 dimensiones que se constituyen como las variables de este estudio y que permitieron la siguiente evaluación:

Primera dimensión: Se denominó trabajo independiente e identificó las concepciones previas de los estudiantes en torno al concepto de trabajo independiente $(6$ preguntas escala Likert y la última de tipo abierto).
1. Segunda dimensión, base para el presente artículo: Se denominó estilos de aprendizaje, para esta se utilizó el cuestionario C.H.A.E.A. (Cuestionario Honey/ Alonso de Estilos de Aprendizaje), que originalmente consta de 80 ítems, y se estructura en cuatro grupos o secciones de veinte preguntas correspondientes a los cuatro estilos de aprendizaje (activo, reflexivo, teórico y pragmático). Todos los ítems están distribuidos aleatoriamente formando un solo conjunto. Sin embargo para el presente estudio en razón a que el instrumento completo era bastante extenso, se revisaron las preguntas de control y se disminuyó el número de ítems a 60, conservando la misma proporción para cada estilo, Se realizó un baremo general de interpretación de resultados de los 4 estilos de aprendizaje para el análisis.

\begin{tabular}{|c|c|c|c|c|}
\hline $\begin{array}{l}\text { Categoría } \\
\text { puntuación }\end{array}$ & $\begin{array}{l}\text { Estilo } \\
\text { Activo }\end{array}$ & Estillo Reflexivo & Estillo Teórico & $\begin{array}{l}\text { Estilo } \\
\text { Pragmático }\end{array}$ \\
\hline Predominancia Baja & Menora 7 & Menor a 10 & Menor a 10 & Menora 9 \\
\hline Predominancia Media & De 7 ag & De 10 a 12 & De 10 a 11 & Dega 11 \\
\hline Predominancia Alta & Mayor a 9 & Mayor a 12 & Mayor a 11 & Mayor a 11 \\
\hline
\end{tabular}

Con relación a este instrumento se puede afirmar que el cuestionario, se basa en las teorías del aprendizaje de tipo cognitivo, los autores de este, coinciden en la definición y el desarrollo del proceso de aprendizaje como un proceso cíclico dividido en cuatro etapas, en las que influye en un alto porcentaje las experiencias vividas, las circunstancias medio-ambientales y 10 heredado.

Este cuestionario fue elegido para el estudio porque ayuda al estudiante a perfeccionar y mejorar el estilo de aprendizaje considerando las preferencias durante el proceso educativo. Así mismo facilita la detección de la forma en que aprenden según el contexto en que se encuentran: 
aula, grupo de trabajo, tutoría, taller o laboratorio, entre otros.

La tercera dimensión: Se denominó autoimagen, está compuesta por diez ítems y permitió revisar las apreciaciones que tienen los estudiantes de ellos mismos con relación a ellos mismos, a como los ven sus compañeros y docentes, de igual modo se incluyó un ítem, que permitió verificar el nivel de satisfacción 0 agrado con el programa académico estudiado.

La cuarta dimensión: Se denominó aprendizaje autónomo, y desarrolló con base en el instrumento CPEI-U Castellanos (2011), permitió evaluar los cuatro aspectos que se consideran primordiales al hablar de aprendizaje autónomo.

De manera complementaria, el instrumento completo recolectó datos específicos de la situación sociodemográfica de cada estudiante encuestado, permitiendo identificar aspectos relacionados con las condiciones de vivienda y configuración familiar además de edad, situación laboral y académica entre otros, datos que al ser analizados de manera cruzada con las demás dimensiones permitieron obtener guías para proyectar la intervención requerida.

Muestreo y proceso de implementación:

Para la recolección de los datos se contabilizaron los estudiantes matriculados en las asignaturas del Componente Proyectual/Investigativo del Programa de Diseño Industrial, lo que permitió trabajar sobre una población finita de 111 estudiantes estratificados por semestre.

Sobre esta población se realizó un muestreo aleatorio simple permitiendo definir en un primer momento el cálculo para el estudio piloto y de manera complementaria el total de la muestra.
El análisis de los datos asociados a la actual investigación involucró una serie de métodos descriptivos univariantes $y$ multivariantes. Con el propósito de formar grupos de ítems en función de las proporciones obtenidas, se utilizó el análisis de clúster con el objeto de formar dos grupos de proporciones, los cuales se ordenaron según la proporción. Finalmente, la codificación de todos los puntajes para las cuatro dimensiones se cruzó con los datos sociodemográficos del cuestionario y mediante un análisis de correspondencias múltiples se discutieron las variables sociodemográficas en términos de los estratos del perfil para cada dimensión.

\section{RESULTADOS Y CONCLUSIONES}

Con relación a la dimensión 1 se identificó, que el $49,47 \%$ de los estudiantes entendían de manera correcta el concepto de trabajo independiente y se evidenció la ejecución de actividades propias para el cumplimiento de este aspecto.

Se pudo entonces concluir que si bien casi el $50 \%$ de los estudiantes que cursan las asignaturas del Componente Proyectual/Investigativo del Programa de Diseño Industrial, entienden de manera acertada el concepto de trabajo independiente, (trabajo que se realiza sin acompañamiento directo del docente para fortalecer los procesos de aprendizaje), es el porcentaje restante el que requiere atención, en razón a que son ellos los que requieren acciones desde lo académicocurricular para nivelar los conceptos básicos que permitan un correcto aprendizaje, más aún si se revisa con detenimiento que estos estudiantes no solo se ubican en los semestres iniciales, lo que deja entrever que han desarrollado las actividades extra-clase sin una comprensión profunda, dificultando un aprendizaje altamente significativo.

También es importante evidenciar que no se identificó ninguna correlación entre esta dimensión y sus preferencias en cuanto a 
estilos de aprendizaje, pero de manera contraria si es evidente que los estudiantes que comprenden el concepto, evidencian una mayor apropiación de estrategias propias de un aprendiz autónomo, independientemente de sus condiciones socio-económicas y culturales.

La dimensión 2, la cual se evaluó bajo una escala dicotómica, permitió identificar que de la muestra el $40 \%$ pertenecen al estilo de aprendizaje reflexivo, el $22 \%$ son teóricos, el $8,2 \%$ son pragmáticos dejando solo el $2,4 \%$ en la categoría predominante de activos (ver tabla 2). De manera paralela se revisaron los resultados en los que un solo individuo manifestaba distintos estilos en grados iguales 0 muy cercanos, permitiendo generar las siguientes asociaciones predominantes:

Teórico- reflexivo con un $8,2 \%$,

Pragmático - reflexivo $7,1 \%$,

Teórico - pragmático 5,9\%, Reflexivo activo $2,4 \%$,

Reflexivo - teórico - pragmático, reflexivo teórico - activo y reflexivo - pragmático activo, $1,2 \%$ cada uno.

Lo anterior permitió evidenciar que tanto en la predominancia única como en las compuestas la preferencia marcada se da hacia el Estilo de Aprendizaje Reflexivo, seguido del Teórico, dejando en un margen considerablemente menor a los estilos Activos y Pragmáticos, que para la presente clasificación son opuestos.

\begin{tabular}{|l|l|l|}
\hline ESTILO & FRECUENCIA & PORCENTAJE \\
\hline Reflexivo & 34 & 40 \\
\hline Teórico & 19 & 22 \\
\hline Pragmático & 7 & 8,2 \\
\hline Activo & 2 & 2,4 \\
\hline Reflexivo Teórico - & 7 & 8,2 \\
\hline Reflexivo Pragmático & 6 & 7,1 \\
\hline Teórico- Pragmático & 5 & 5,9 \\
\hline Reflexivo- Activo & 2 & 2,4 \\
\hline & & \\
\hline Reflexivo -Teórico - pragmático & 1 & 1,2 \\
\hline Reflexivo - Teórico - activo & 1 & 1,2 \\
\hline Reflexivo - Pragmático - activo & 1 & 1,2 \\
\hline TOTAL & 85 & 100 \\
\hline
\end{tabular}

Tabla 2. Distribución de frecuencias y porcentajes para los estilos encontrados según los puntajes obtenidos del cuestionario. resultados de la tabla obtenidos por autores.
Al correlacionar la información con las otras dimensiones de la investigación, se evidenció que existe relación significativa entre los estilos de pensamiento Pragmático, Reflexivo, Activo, Teórico, Teórico-Reflexivo con estudiantes que se encuentran en $3^{\circ}$ y $5^{\circ}$ a $10^{\circ}$ semestre, cuyas edades están entre 19 a 31 años, con promedios acumulados entre 3.0 y 4.0 y su estado civil soltero.

De otro lado, se encuentra evidencia de la relación (con menos fuerza) entre los estilos Reflexivo-Activo, Teórico -Reflexivo -Activo, Pragmático -Activo -Reflexivo con estudiantes de $4^{\circ}$ semestre, con promedios entre 4.1 y 4.5 . Además también existe relación entre el estilo Teórico -Pragmático y estudiantes con edades entre 15 y 18 años, que se encuentran entre primer y segundo semestre, en cuanto al promedio acumulado no dan información.

De manera complementaria, el análisis permitió inferir la relación entre las estrategias pedagógicas más utilizadas al interior del Programa (carácter investigativo y educación por proyectos) y las predominancias evidenciadas, dado que según las teorías expuestas por Baldomero, L. (2008) citado por Gravini, M., Cabrera, E., Ávila, V., Vargas, I., (2009), estas favorecen los estilos identificados.

\begin{tabular}{l|l}
\hline Estilos de aprendizaje del estudiante & Estrategias \\
\hline Activo & $\begin{array}{l}\text { Foro, sociodrama, preguntas intercaladas } \\
\text { yproyectos }\end{array}$ \\
\hline Reellexivo & Estudio de casos, ensayos y resumen \\
\hline Pragmático & $\begin{array}{l}\text { Ilsstraciones, lluvia de ideas y taller } \\
\text { educativo. }\end{array}$ \\
\hline Teórico & $\begin{array}{c}\text { Mapas conceptuales, seminario, relatorias } \\
\text { yresumen. }\end{array}$ \\
\hline
\end{tabular}

Tabla 3. Estrategias propuestas para la activación de cada estilo, resultados de la tabla obtenidos por autores, (Basado en Gravini et all, 2009).

En resumen se puede concluir que existe concordancia en la manera de aproximarse al conocimiento por parte de los estudiantes que muestran una preferencia por el estilos reflexivo (mayoría) y las actitudes comúnmente observadas y descritas por los 
docentes, es decir: el estilo reflexivo se aproxima al conocimiento a través de la recolección de datos mediante la observación la cual es comparada con otros puntos de vista, lo que permite un análisis detallado de la situación, procurando la lógica racional y objetiva, sin embargo tienden a ser menos prácticos y dilatar la proyección real de sus ideas.

Lo anterior se reconfirma al revisar que el estilo que presenta menor frecuencia es el activo, quienes se caracterizan por evidenciar que aprenden resolviendo problemas, trabajando en equipo y viviendo nuevas experiencias, características menos observadas en el grupo estudiado.

También otros autores como Lago, B., Colvin, L. \& Cacheiro, M. (2008) citados por (Lago et al., 2008, p.13) en su artículo Estilos de Aprendizaje y Actividades Polifásicas: Modelo EAAP basan su modelo dándole una utilidad pedagógica a la hora de seleccionar las estrategias de Enseñanza-Aprendizaje sobre los estilos de aprendizaje.

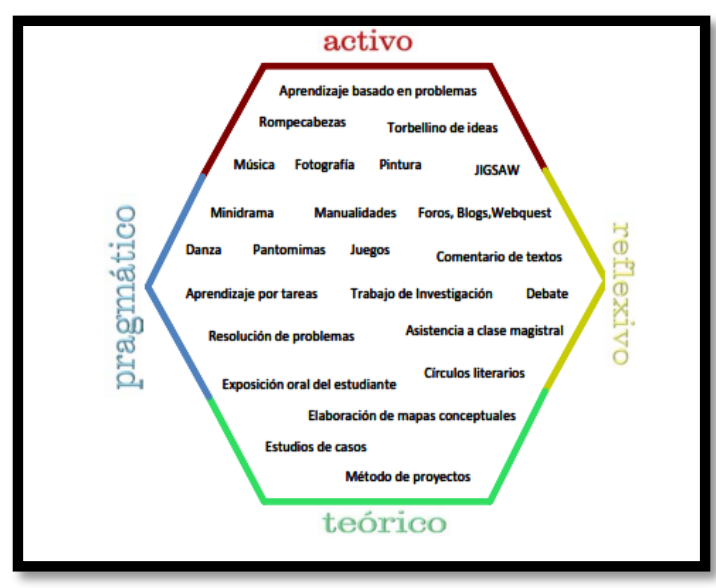

Figura 1: Repertorio de Actividades de enseñanza-aprendizaje Lago et al., 2008 (p.17)

Es importante resaltar que al analizar los datos de manera detallada se pudo encontrar una constante interesante en relación con los estudiantes que presentaron mayor nivel académico, si bien no se puede considerar una muestra representativa, pues los casos son solo 4 , si
Ilama la atención que para estos estudiantes independientemente del estilo preferido, es notoria la predominancia alta en el estilo activo, el cual en la muestra total es el más bajo.

Para la dimensión 3 se concluye que: la mayoría de los estudiantes manifiestan tener una auto imagen favorable, y un número considerablemente menor evidencian lo contrario (baja autoimagen), sin embargo esta última puede estar interfiriendo en su desarrollo académico, pues al estar relacionada con la cuarta dimensión afecta la implementación de estrategias propias de aprendizaje autónomo.

Para la dimensión 4 se identificó que los estudiantes tienen una actitud positiva ante el estudio y están de acuerdo en plantear paso a paso qué hacer para conseguir una "ejecución eficaz" en concordancia con las actividades de control estratégico y personal, que les permitan posteriormente evaluar y supervisar dicha eficacia de los pasos seguidos; en cuanto a la selección y uso de estrategias están de acuerdo con seleccionar las estrategias cognitivas más acordes con la consecución de sus objetivos y la realización de un plan estratégico pero desconocen la preferencia de su propio estilo de aprendizaje lo que les genera mayor desgaste, pues solo por prueba y error identifican las que más les son efectivas. Para la evaluación de estrategias específicas del meta-conocimiento (saber cómo, cuándo, por qué usar una estrategia específica y observar la eficacia de ella para cambiarla de ser necesario y controlar los distractores existentes) evidencian requerir mayor apoyo, lo que confirma que no conocer de manera particular sus preferencias en estilos de aprendizaje dificulta la implementación de las estrategias que propician el aprendizaje autónomo.

Para terminar, el análisis de la tercera y cuarta dimensión permitió concluir que el 
uso de estrategias por parte de los estudiantes objeto de estudio durante el desarrollo de sus actividades de trabajo independiente se encuentra clasificado en un nivel medio, resaltándose la tendencia al nivel superior de aquellas que refieren la actitud positiva ante el estudio.

\section{REFERENCIAS BIBLIOGRÁFICAS}

Alonso, C.M. (2001). Estilos de aprendizaje. Presente y futuro. Estilos de aprendizaje. (1)1. 4-15.

Castellanos S., Palacio M., Cuesta M., García E., (2011). Cuestionario de Evaluación del Procesamiento Estratégico de la Información para Universitarios (CPEIU). Revista Electrónica de Metodología Aplicada, 16 (2), 15-28.

Gallego D., Alonso C., (2008). Estilos de aprendizaje en el siglo XXI. Revista Estilos de Aprendizaje, 2 (2), 23-34.

Gravini, M., Cabrera, E., Ávila, V., Vargas, I., (2009). Estrategias de enseñanza en docentes y estilos de aprendizaje en estudiante del programa de psicología de la universidad Simón Bolívar, Barranquilla. Revista Estilos de Aprendizaje, 3 (3), 124139.

lafrancesco, G. (2012). Aprendizaje Autónomo y Cognición. Bogotá, Colombia: CORIPET.

Lago, B., Colvin, L., Cacheiro, M., (2008). Estilos de Aprendizaje y Actividades Polifásicas: Modelo EAAP. Revista Estilos de Aprendizaje, 2 (2), 1-22.León, G. (2004). La educación en el contexto de la globalización. Rhela.vol 6,343-354.

Ministerio de Educacion Nacional de Colombia, (2009). Educación superior. Recuperado de: http://www.mineducacion.gov.co/1621/article $-196477 . h t m l$
Rubia, M. y Marbán, J.Mª (2006). E papel de las nuevas tecnologías en el desarrollo de proyectos piloto de innovación docente, Revista Latinoamericana de Tecnología Educativa, 5 (2), 301-308. Recuperado de:http://www.unex.es/didactica/RELATE C/ sumario_5_2.htm 